\title{
CHEBYSHEV ESTIMATES FOR BEURLING GENERALIZED PRIME NUMBERS
}

\author{
HAROLD G. DIAMOND ${ }^{1}$
}

Abstract. We consider a Beurling generalized number system for which the counting function of the integers satisfies

$$
N(x)=c x+O\left(x \log ^{-\gamma} x\right)
$$

for some positive $c$ and $\gamma$. Beurling showed that the prime number theorem must hold if $\gamma>\frac{3}{2}$, but it can fail to hold if $\gamma \leqq \frac{3}{2}$. It was shown by Hall in the preceding article that the Chebyshev prime counting estimates

$$
0<\liminf _{x \rightarrow \infty} \frac{\psi(x)}{x} ; \quad \limsup _{x \rightarrow \infty} \frac{\psi(x)}{x}<\infty
$$

can fail if $\gamma<1$. Here we shall prove that these estimates hold for any system satisfying $\gamma>1$. The proof uses a convolution approximate inverse of the measure $d N$.

Chebyshev established the correct order of magnitude of the weighted prime counting function $\psi$ by exhibiting numbers $a>0$ and $A<\infty$ such that

$$
\liminf _{x \rightarrow \infty} \frac{\psi(x)}{x} \geqq a, \quad \limsup _{x \rightarrow \infty} \frac{\psi(x)}{x} \leqq A .
$$

The prime number theorem (P.N.T.) asserts that $a=A=1$. Here we shall study Chebyshev estimates for Beurling generalized prime numbers.

The first paragraph of the preceding paper by Hall mentions the basic facts about Beurling generalized numbers. For a more detailed discussion, see e.g. [1] or [2]. The generalized psi function is defined on $[1, \infty)$ by setting

$$
\psi(x)=\sum_{p_{i}^{\alpha} \leqq x} \log p_{i}
$$

where $p_{1}, p_{2}, p_{3}, \cdots$ is the sequence $P$ of generalized primes and $\alpha$ ranges over positive integers. All other notation is as in Hall's paper.

Received by the editors November 13, 1972.

AMS (MOS) subject classifications (1970). Primary $10 \mathrm{H} 40$.

${ }^{1}$ Research supported in part by NSF grant GP-28920.

(C) American Mathematical Society 1973 
Beurling [2] proved that if

$$
N(x)=c x+O\left(x \log ^{-\gamma} x\right)
$$

for some $c>0$ and $\gamma>\frac{3}{2}$, then $\psi(x) \sim x$, i.e., the P.N.T. holds for $P$. Also, he showed by example that the P.N.T. could fail for $\gamma \leqq \frac{3}{2}$. In the preceding article and in [5] Hall proves that the Chebyshev estimates can fail for $\gamma<1$. In the present paper we study the remaining range $1<\gamma \leqq \frac{3}{2}$ and prove the following

THEOREM. If $\boldsymbol{P}$ is a generalized prime number system for which $N_{P}$ satisfies (2) with $c>0$ and $\gamma>1$, then there exist numbers $a>0$ and $A<\infty$ for which (1) holds.

In [1] Bateman and the author had conjuctured that the upper Chebyshev estimate holds when $\gamma>1$. A similar conjecture was recently mentioned to the author by Professor J. H. Chalk. This estimate has applications to tauberian theorems of T. M. K. Davison [3] and S. L. Segal [6].

We begin by proving the upper estimate; the lower estimate then follows easily. The method of Chebyshev, which exploits the relation

$$
\psi(2 x)-\psi(x)+\sum_{j=1}^{\infty}\left\{\psi\left(\frac{x}{j+1 / 2}\right)-\psi\left(\frac{x}{j+1}\right)\right\}=2 x \log 2+O(\log x)
$$

appears to be of no use in the present situation.

Let $F:[1, \infty) \rightarrow \boldsymbol{R}$ be of bounded variation on each interval $[1, x]$. There is an associated Borel-Stieltjes measure on $[1, \infty)$ which we denote by $d F$. We define an operator $L$ on measures by setting

$$
\int_{E} L d F=\int_{t \in E} \log t d F(t)
$$

Also, we employ multiplicative convolution of measures, denoted by $*$. The starting point of our proof is the Chebyshev formula $\sum_{d \mid n} \Lambda(d)=\log n$ which, in the form

$$
d \psi * d N=L d N
$$

is valid for Beurling generalized numbers (see [4]). We convolve each side of (3) by $\left(\delta-\varepsilon t^{-\varepsilon} d t\right) *(\log e t)^{-\gamma} d t$, where $\delta$ denotes Dirac measure at 1 , $d t$ is Borel-Lebesgue measure on $[1, \infty)$ and $\varepsilon$ is a positive number presently to be specified. We shall show that the new factors act like an approximate convolution inverse of $d N$. Let

$$
v_{\varepsilon}(x):=\int_{1}^{x} d N *\left(\delta-\varepsilon t^{-\varepsilon} d t\right) *(\log e t)^{-\gamma} d t .
$$


Lemma 1. Assume that $N$ satisfies (2) with $c>0$ and $\gamma>1$. There exists a positive number $\varepsilon$, depending on $N$, such that $\nu_{\varepsilon}(x) \geqq 0$ for all $x \geqq 1$ and $\nu_{\varepsilon}(x) \rightarrow \infty$ as $x \rightarrow \infty$ :

Proof. We write

$$
N(x)=c x+\theta K x(\log e x)^{-\gamma}
$$

where we may suppose that $1<\gamma<2, K>0$ and $|\theta| \leqq 1$. Let

$$
\varepsilon=\exp \{-16 e K / c(\gamma-1)\}
$$

If $1 \leqq x \leqq 1 / \varepsilon$, then

$$
\int_{1}^{x} d N *\left(\delta-\varepsilon t^{-\varepsilon} d t\right) \geqq N(x)-\varepsilon N(x)(x-1) \geqq 0 .
$$

The lemma is certainly true for $1 \leqq x \leqq 1 / \varepsilon$ since the third convolution factor is everywhere nonnegative.

If $x>1 / \varepsilon$, we utilize all the convolution factors. We write

$$
\begin{aligned}
v_{\varepsilon}(x)= & c \int_{1}^{x}(\delta+d t) *\left(\delta-\varepsilon t^{-\varepsilon} d t\right) *(\log e t)^{-\gamma} d t \\
& +\int_{1}^{x}(d N-c \delta-c d t) *\left(\delta-\varepsilon t^{-\varepsilon} d t\right) *(\log e t)^{-\gamma} d t \\
= & I_{1}+I_{2}, \text { say. }
\end{aligned}
$$

We evaluate $I_{1}$ by iterated integration, first combining the first and second factors and then changing the integration variable. We find that

$$
I_{1}=c x \int_{1}^{x} t^{-\varepsilon-1}(\log e x / t)^{-\gamma} d t .
$$

To estimate $\left|I_{2}\right|$ let

$$
\begin{gathered}
d \alpha(t)=\delta+\varepsilon t^{-\varepsilon} d t \text { and } d \beta(t)=(\log e t)^{-\gamma} d t \\
\left|I_{2}\right| \leqq \int_{1}^{x} K x t^{-1}(\log e x / t)^{-\gamma}(d \alpha * d \beta)(t) .
\end{gathered}
$$

We again iterate the integration, obtaining first

$$
\begin{aligned}
\int_{1}^{x} K x t^{-1}(\log e x / t)^{-\gamma} d \beta(t) \leqq & \int_{1}^{\sqrt{ } x} K x(\log e \sqrt{ } x)^{-\gamma} t^{-1}(\log e t)^{-\gamma} d t \\
& +\int_{\sqrt{x}_{x}}^{x} K x(\log e x / t)^{-\gamma} t^{-1}(\log e \sqrt{ } x)^{-\gamma} d t \\
\leqq & 8 K x(\log e x)^{-\gamma} /(\gamma-1) .
\end{aligned}
$$


Then

$$
\begin{aligned}
\left|I_{2}\right| & \leqq \frac{8 K}{\gamma-1} \int_{1}^{x} \frac{x}{t}\left(\log \frac{e x}{t}\right)^{-\gamma}\left(\delta+\varepsilon t^{-\varepsilon} d t\right) \\
& =\frac{8 K x}{\gamma-1}(\log e x)^{-\gamma}+\frac{8 K \varepsilon x}{\gamma-1} \int_{1}^{x} t^{-\varepsilon-1}\left(\log \frac{e x}{t}\right)^{-\gamma} d t .
\end{aligned}
$$

Now $8 K \varepsilon /(\gamma-1)<c / 3$ by the choice of $\varepsilon$. Next, we show that for $x>1 / \varepsilon$

$$
\frac{8 K x}{\gamma-1}(\log e x)^{-\gamma}<\frac{1}{2} c x \int_{1}^{x} t^{-\varepsilon-1}(\log e x / t)^{-\gamma} d t .
$$

Indeed, we have

$$
\begin{aligned}
\frac{1}{2} c x \int_{1}^{x} t^{-\varepsilon-1}(\log e x / t)^{-\gamma} d t & >\frac{1}{2} c x(\log e x)^{-\gamma} \int_{1}^{1 / \varepsilon} t^{-\varepsilon-1} d t \\
& >\frac{c}{2} x \varepsilon^{\varepsilon}\left(\log \frac{1}{\varepsilon}\right)(\log e x)^{-\gamma}>\frac{8 K x}{\gamma-1}(\log e x)^{-\gamma}
\end{aligned}
$$

since $\varepsilon^{e} \geqq \exp (-1 / e)$ for $0<\varepsilon<1$. Now

$$
v_{\varepsilon}(x) \geqq I_{1}-\left|I_{2}\right| \geqq \frac{1}{6} c x \int_{1}^{x} t^{-\varepsilon-1}(\log e x / t)^{-\gamma} d t .
$$

The last term is clearly positive and tends to infinity with $x$.

LEMMA 2.

$$
\int_{1}^{x} L d N *\left(\delta-\varepsilon t^{-\varepsilon} d t\right) *(\log e t)^{-\gamma} d t=O(x) .
$$

Proof. We apply iterated integration. First

$$
\int_{1}^{x} L d N *\left(\delta-\varepsilon t^{-\varepsilon} d t\right)=c x / \varepsilon+o(x)=O(x) .
$$

Next,

$$
\int_{1}^{x} O\left(\frac{x}{t}\right)(\log e t)^{-\gamma} d t=O(x) .
$$

Próof OF THE UPPER estimate. Formula (3) yields

$$
\begin{aligned}
\int_{1}^{x} d N *\left(\delta-\varepsilon t^{-\varepsilon} d t\right) * \frac{d t}{(\log e t)^{\gamma}} * d \psi \\
=\int_{1}^{x} L d N *\left(\delta-\varepsilon t^{-\varepsilon} d t\right) * \frac{d t}{(\log e t)^{\gamma}} .
\end{aligned}
$$


The right-hand side of this equation was just shown to be $O(x)$. The left side is representable as $\int_{1}^{x} v_{\varepsilon}(x / t) d \psi(t)$. We have shown that $v_{\varepsilon}(u) \geqq 0$ for all $u \geqq 1$ and that there exists a number $B$ for which $\nu_{\varepsilon}(u) \geqq 1$ for all $u \geqq B$. Also $\psi$ is an increasing function. Thus we have

$$
\psi(x / B) \leqq \int_{1}^{x} v_{\varepsilon}(x / t) d \psi(t)=O(x),
$$

or $\lim \sup _{x \rightarrow \infty} \psi(x) / x<\infty$.

PROOF OF THE LOWER ESTIMATE. Easy calculations show that

$$
\begin{aligned}
\rho(x):=\int_{1}^{x} d N *\left(\delta-\frac{d t}{t}\right) & =O\left\{x(\log e x)^{-\gamma}\right\}, \\
\int_{1}^{x} L d N *\left(\delta-\frac{d t}{t}\right) & =c x+O\left\{x(\log e x)^{1-\gamma}\right\} .
\end{aligned}
$$

We convolve both sides of (3) by $\delta-t^{-1} d t$ and use the last estimates. We have

$$
\begin{aligned}
c x+O\left(x \log ^{1-\gamma} x\right) & =\int_{1}^{x} \rho(x / t) d \psi(t) \\
& \leqq K \int_{1}^{x} \frac{x}{t}\left(\log \frac{e x}{t}\right)^{-\gamma} d \psi(t) .
\end{aligned}
$$

Integration by parts and omission of a negative term yield

$$
c x+o(x) \leqq K\left\{\psi(x)+\int_{1}^{x} \psi\left(\frac{x}{u}\right)(\log e u)^{-\gamma} d u\right\} .
$$

By the upper estimate for $\psi$ there exists a number $M$ such that $\psi(x) \leqq$ $M x$ for all positive $x$. Now choose $B \geqq 1$ so large that

$$
K M \int_{B}^{\infty} \frac{x}{u}(\log e u)^{-\gamma} d u<\frac{1}{3} c x .
$$

If $x$ is large enough so that $o(x)>-(1 / 3) c x$, then we have

$$
\frac{1}{3} c x<K \psi(x)+K \psi(x) \int_{1}^{B}(\log e u)^{-\gamma} d u \leqq K B \psi(x) .
$$

Consequently,

$$
\liminf _{x \rightarrow \infty} \psi(x) / x \geqq \frac{c}{3 K B}>0 .
$$




\section{REFERENCES}

1. P. T. Bateman and H. G. Diamond, Asymptotic distribution of Beurling's generalized prime numbers, Studies in Number Theory, vol. 6, Math. Assoc. Amer., PrenticeHall, Englewood Cliffs, N.J., 1969, pp. 152-210. MR 39 \#4105.

2. A. Beurling, Analyse de la loi asymptotique de la distribution des nombres premiers généralisés. I, Acta Math. 68 (1937), 255-291.

3. T. M. K. Davison, A Tauberian theorem and analogues of the prime number theorem, Canad. J. Math. 20 (1968), 362-367. MR 37 \#168.

4. H. G. Diamond, Asymptotic distribution of Beurling's generalized integers, Illinois J. Math. 14 (1970), 12-28. MR 40 \#5555.

5. R. S. Hall, Theorems about Beurling's generalized primes and the associated zeta function, Ph.D. Thesis, University of Illinois, Urbana, Ill., 1967.

6. S. L. Segal, A Tauberian theorem for Dirichlet convolutions, Illinois J. Math. 13 (1969), 316-320. MR 39 \#143.

Department of Mathematics, University of Illinois, Urbana, Illinois 61801 